Rev. Chil. Pediatr. 60 (1); 18-23, 1989

\title{
Lateralidad manual, disfunción cerebral y dislexia
}

\author{
Dra. Amanda Céspedes C. ${ }^{1}$; Psic. Jaime Berneosolo B. ${ }^{2}$; Psic. Lujs Bravo V. ${ }^{3}$;
} St. Arturo Pinto G, ${ }^{4}$

\section{Handedness in children with and with out dyslexia}

A frequency distribution of handedness and its relations with minor signs of neurological dysfunction was studied in a group of 56 dyslexic children and 56 good readers. An unusual frequency of $18 \%$ left-handed children in the dyslexic group and the high frequency of soft signs in this lefthanded children, is in concordance with recent hypothesis about the dysgenesic brain origin of dyslexia and leftlandedness, postulated by Galaburda, Geschwind and others. They suggest a distorted cortical development of the brain areas related with linguistic functions, with subsequent expression on verbal language reading ability and handedness.

(Key words: handedness, cerebral dysfunction and reading disability.)

1. Docente e Investigador Departamento de Educación Especial, Facultad de Educación, Universidad Ca. tólica de Chile.

2. Psicólogo. Docente e Investigador Departamento de Educación Especial, Universidad Católica de Chile.

3. Psicólogo. Director del Departamento de Educación Especial, Universidad Católica de Chile.

4. Profesor Magister en Educación Especial, Departamento de Educación Especjal, Universidad Católica de Chile.

Esta investigación se llevó a cabo con ayuda de la Dirección de Investigación de la Universidad Católica de Chile (DIUC) y del Consejo Superior de Ciencias
La existencia de una alteración en la organización funcional cerebral como sustrato de la dislexia infantil ha sido planteada por diversas teorias, a partir de los postulados de Samuel Orton $^{1}$ acerca de la relación entre dominancia cerebral y lateralidad periférica. Recientemente el origen disgenésico de la dislexia ha vuelto a

(CONICYT) y presentado por la Dra. Amanda Céspedes como Trabajo de lncorporación a la Sociedad Chilena de Psiquiatría, Neurología y Neurocirugía en diciembre de 1985 . 
cobrar relevancia, con los hallazgos de Galaburda, Geschwind y otros, respecto a anormalidades citoarquitectónicas corticales cerebrales, zurdera $y$ dislexia ${ }^{2,3}$.

Si bien alrededor de los dos anos de edad ya está fuertemente establecida en el niño una preferencia manual en la ejecución de diversas destrezas, tales como lanzar, manipular utensilios o ejercitar actividades constructivas, esta preferencia no es un predictor de la manualidad definitiva, la cual se considera firmemente establecida sólo después de los 8 años de edad. En la práctica, la condición de diestro o zurdo se adjudica según la mano que se emplea para escribir ${ }^{4}$.

En la manualidad humana convergerían dos características relacionadas, pero conceptualmen. te diferentes: la manualidad de un individuo -va. le decir, su condición de diestro o zurdo-y la tendencia diestra de la población humana. Según Anett ${ }^{\text {5-7 }}$ habría una influencia genética sobre la distribucjón al azar de la manualidad. Este gen (o genes) determinaría la asimetría anatómica cerebral que subyace a la dominancia hemisférica para el lenguaje verbal. El hemisferio izquierdo sería superior en la programación de eventos organizados serialmente y finamente secuenciados, entre los cuales estaría el lenguaje verbal proposicional y el lenguaje escrito, además de un sinnúmero de habilidades práxicas manuales ${ }^{8}$. Si la manualidad fuese un fenómeno exclusivamente genético, la distribución de diestros homocigotos, diestros heterocigotos y zurdos homocigotos sería binomial: en 100 sujetos habría: $\left(8^{2}+(2 \times 8 \times 2)+2^{2}\right)=64$ diestros homocigotos, 4 zurdos homocigotos y 32 diestros heterocigotos, lo cual daría 2 fenotipos: 96\% de diestros y $4 \%$ de zurdos.

Sin embargo, las diferencias individuales en la manualidad surgen independientes del genotipo; sobre ellas gravitan otros factores, de los cuales cabe destacar aquellos de índole sociocultural ("zurdos contrariados", por ejemplo) $y$ los factores adversos ambientales tempranos pre y perinatales que puedan afectar el desarrollo cerebral ${ }^{\mathrm{B}}, 9$. En consecuencia, si bien la población humana muestra una tenđencia diestra, desde el punto de vista más indivicual es posjble identificar subgrupos de lateralidad manual, cuyo tipo y frecuencia va a depender del universo a considerar y de la edad de los sujetos de dj. cho universo.
En esta perspectiva se sitúan los numerosos estudios sobre zurdera, entre los cuales cabe destacar a Satz y su descripción de un sindrome de Zurdería Patológica en niños y adultos ${ }^{10}$, 11 y a Geschwind, quien encuentra que los trastornos inmunológicos son 3 veces más frecuentes en zurdos que en diestros, la migraña es 2 veces más frecuente y los trastornos específicos del aprendizaje se dan en $10 \%$ de zurdos y sólo en $2 \%$ de diestros ${ }^{12}$.

Entre escolares la frecuencia de zurdera es significativamente mayor en disléxicos que en niños que leen normalmente: $19 \%$ de varones y $15 \%$ de niñas zurdos en un grupo de disléxicos mayores de 8 anos, mientras que en lectores normales la frecuencia de zurdería fue de $13,6 \%$ en varones y $5,5 \%$ en nifias ${ }^{14}$.

En el presente trabajo se estudió la existencia de subgrupos de lateralidad manual en un grupo de escolares disléxicos comparado con un grupo de lectores normales $y$ se intentó relacionar el tipo de lateralidad con el grado aparente de disfunción cerebral, medida a través de los denominados "signos menores" de disfunción neurológica ("soft signs" de la literatura anglosajona).

\section{MATERIAL Y METODO}

La muestra experimental estuvo constituida por 56 escolares de ambos sexos que curnplían con los siguientes requisitos:

Edad entre 8 y 12 años; nivel socioeconómico medio bajo (padres obreros o empleados subalternos); inteligencia normal lenta a superior (C.I. superior a 85 en el test Wisc-r); escolaridad superior a 2 años; asistencia regular a los grupos diferenciales en sus escuelas; ausencia de alteraciones sensoriales o motoras severas (evaluación neuropediátrica de descarte); lectura inícial, con un nivel lector inferior al percentil 30 de la escala TEDE de Condemarín-Blomquist.

El grupo de lectores normales estuwo constituido por 56 niños de ambos sexos y similar rango etario, que provenían de las mismas escuelas y aulas que los niños disléxioos, con inteligencia normal lenta a superior y un nivel lector normal (percentil 40 o superior en la prueba TEDE).

Las pruebas apicadas a ambos grupos consistieron en: pauta de evaluación de la lateralidad periférica, constituida por un protocolo de destrezas medidas directamente y un cuestionario de destrezas no medidas en forma directa. Esta pauta se elaboró sobre la base de evaluaciones previamente validadas y contenía los siguientes ítemes: preferencia de la mano en escribir, dibujar, borrar con goma, abrir un candado, enhebrar una aguja. Preferencia del pie en patear la pelota, saltar en un pie, golpear con un pie, coger una bolita 
entre los ortejos. Preferencia del ojo en mirar a través de un agujero, mirar a través del ojo de la cerradura, mirar al interior de una botella, hacer un guifio. Preferencia del oido en escuchar una conversación a través de la puerta cerrada, escuchar el tic-tac de un reloj pulsera, colocarse un audífono de radio, escuchar los latidos del corazón de otra persona. Cuestionario: ¿Con qué mano usas el cepillo de dientes?, ¿la peineta?, ¿el pañuelo?, ¿con qué pie bajas de la micro?, ¿subes un peldaño?, ¿apagas un cigarrillo o aplastas a una araña?, ¿cómo hablas por teléfono?, ¿cótno apuntas con un rifle?

Protocolo de examen neurológico, basado en el examen clínico propuesto por Peters y col. ${ }^{15}$, extractándose 25 ítemes, agrupados en: habilidad motriz grues; habilidad motriz fina; motricidad extraocular; motricidad facial $y$ praxias orolinguales; discriminación derecha-izquierda; signos neurológicos anornales especificos (sincinesias, alteración de los reflejos profundos, movimientos coreiformes).

La cuantificación de los datos obtenidos mediante este protocolo permitió establecer 4 categor ías:

0 : Sin signos de disfunción neurobógica $(0$ a 5 signos menores)

1: Disfunción neurológica leve (6 a 10 signos menores)

2: Disfunción neurológica moderada (11 a 15 signos menores)

3: Disfunción neturológica severa (más de 15 signos menores).

Un solo profesional (A.C.C.) aplicó a ambos grupos los instrumentos de medición, desconociendo a qué grupo pertenecía el nirio exammado. Se aplicó un análisis estadístico de significancia. No se efectuó un anílisis por sexo, debido a lo reducido de las mues. tras.

\section{RESULTADOS}

La aplicación de ja pauta de evaluación de la manualidad y de la preferencia del pie, ojo y oido permitió distinguir 5 subgrupos:

Diestro homogéneo (DH), niños que mostraron una neta manualidad derecha y una fuerte consistencia entre la preferencia derecha del pie, ojo y oido.

Zurdo homogéneo ( $\mathrm{ZH})$, niños que mostra. ron una neta manualidad izquierda y una fuerte consistencia entre la preferencia izquierda del pie, ojo y oído.

Mix to "A" (MA), niños que mostraron una manualidad derecha menos neta (podían realizar algunas praxias manuales con la mano contralateral) y no había una consistencia entre la preferencia del pie, ojo y oído; algunos mostraron mano-pie concordantes, con ojo-oído preferentes contralaterales; otros mano-pie-ojo, concordantes y oido contralateral; mano-pie-oido concordantes con ojo contralateral, o manoojo-oido concordantes, con pie contralateral.

Mix to "B" (MB), ninos que mostraron una clara manualidad izquierda, con preferencia contralateral para pie, ojo y oído.

Ambidjestro (A), ninos que mostraron una débil preferencia manual y una fuerte indefinición en la preferencia del pie y de los órganos sensoriales (ojo y oído).

Cada uno de los $S$ subgrupos mostró un comportamiento caracteristico, que fue evidente en cada uno de los sujetos que componian cada subgrupo. Así, los subgrupos diestro homogéneo (DH) y zurdo homogéneo (ZH) se caracterizaron por la torpeza de la mano no preferente en todo tipo de destrezas manuales, lo cual les llevaba sistemáticamente a transferir el objeto de mano, negando la posibilidad de ejecutar la tarea con la mano no preferente. El subgrupo mix to " $A$ " (MA), en cambio, se caracterizó por mostrar una relativa habilidad práxica con ambas manos, pero con una clara inclinación por elegir la mano preferente derecha. El subgnupo mixto "B" (MB) se caracterizó por la sistemática manualidad izquierda en la habilidad grafomotriz, la tendencia a realizar el resto de las praxias con la mano contralateral y la preferencia derecha del pie, ojo y oído. El grupo Ambidiestro (A) se caracterizó por la gran torpeza de ambas manos en la realización de todo tipo de praxias manuales, con la consiguiente tendencia a transferir una y otra vez el objeto, en un intento por mejorar el desempeño.

La tabla 1 presenta la distribución de las muestras experimental y control, según los subgrupos de lateralidad. Se observa una diferencia significativa entre el porcentaje de $\mathrm{DH}$, en el grupo disléxico en relación al gnupo lector normal, y la presencia de $19 \%$ de niños MB y A en el grupo disléxico; estas categorías no se observan en el grupo lector normal.

La tabla 2 muestra una diferencia estadisticamente significativa entre ambos grupos (disléxico y lector normal) con respecto a la presencia de signos menores de disfunción neurológica: $50 \%$ de niños disléxicos mostraron más de 10 signos menores de disfunción cerebral, mientras que éstos estuvieron presentes sólo en $14,3 \%$ de lectores normales.

La tabla 3 muestra una correlación entre subgrupos de lateralidad, signos menores de disfunción cerebral y dislexia: $61,5 \%$ de los niños disléxicos con disfunción neurológica leve a 
Tabla I

Distribución según lateralidad

\begin{tabular}{|c|c|c|c|c|c|}
\hline \multirow[t]{2}{*}{ Lateralidad } & \multicolumn{2}{|c|}{ Disléxicos } & \multicolumn{2}{|c|}{ Normales } & \\
\hline & No & $\%$ & No & $\%$ & \\
\hline Diestros homogéneos & 35 & 62,5 & 47 & 83,9 & $\mathrm{p}<0,001$ \\
\hline Zurdos homogéneos & 4 & 7,1 & 1 & 1,8 & \\
\hline Mixtos A & 6 & 10,8 & 8 & 14,3 & \\
\hline Mixtos B & 7 & 12,5 & - & - & \\
\hline Ambidiestros & 4 & 7,1 & - & - & \\
\hline TOTAL & 56 & 100,0 & 56 & 100,0 & \\
\hline
\end{tabular}

Tabla 2

Distribución según presencia de signos de anormalidad neucológica

\begin{tabular}{|c|c|c|c|c|c|c|}
\hline \multirow[t]{2}{*}{ Signos neurológicos } & \multicolumn{2}{|c|}{ Disléxicos } & & \multicolumn{2}{|c|}{ Normales } & \\
\hline & $\mathrm{N}^{\mathrm{O}}$ & $\%$ & & $N^{Q}$ & $\%$ & \\
\hline o & 28 & 50 & & 48 & 85,72 & \\
\hline 1 & 14 & 25 & $=0$ & 4 & 7,14 & $1028<<001$ \\
\hline 2 y 3 & 14 & 25 & 30 & 4 & 7,14 & $14,28 \mathrm{p}>0,001$ \\
\hline TOTAL & 56 & 100 & & 56 & 100,0 & \\
\hline
\end{tabular}

Tabla 3

Relación entre lateralidad y signos menores de anormalidad neurológica

\begin{tabular}{|c|c|c|c|c|c|c|c|c|c|c|c|c|c|}
\hline \multirow[t]{4}{*}{ Lateralidad } & \multicolumn{13}{|c|}{ Signos Neurológicos } \\
\hline & \multicolumn{4}{|c|}{ Categoría 0} & \multicolumn{4}{|c|}{ Categoría 1} & \multicolumn{4}{|c|}{ Categorías 2 y 3} & \\
\hline & \multicolumn{2}{|c|}{ Disléxicos } & \multicolumn{2}{|c|}{ Normales } & \multicolumn{2}{|c|}{ Disléxicos } & \multicolumn{2}{|c|}{ Normales } & \multicolumn{2}{|c|}{ Disléxicos } & \multicolumn{2}{|c|}{ Normales } & \\
\hline & No & $\%$ & No & $\%$ & No & $\%$ & No & $\%$ & No & $\%$ & No & $\alpha$ & \\
\hline Diestros homogéneos & 20 & 71,4 & 42 & 87,5 & 8 & 57,1 & 2 & 50,0 & 7 & 50,0 & 3 & 75,0 & \\
\hline Zurdos homogéneos & 4 & 14,3 & 1 & 2,0 & - & - & - & - & - & - & - & - & \\
\hline Mixtos A & 3 & 10,7 & 5 & 10,5 & 2 & 14,3 & 2 & 50,0 & 1 & 7,1 & 1 & 25,0 & \\
\hline Mixtos B & 1 & 3,6 & - & - & 2 & 14,3 & - & - & 4 & 28,6 & - & & $0<0,001$ \\
\hline Ambidiestros & - & - & - & - & 2 & 14,3 & - & - & 2 & 14,3 & - & & $p<0,001$ \\
\hline TOTAL & 28 & 100,0 & 48 & 100,0 & 14 & 100,0 & 4 & 100,0 & 14 & 100,0 & 4 & 100,0 & \\
\hline
\end{tabular}


severa era MB y A, mientras que los lectores normales con signos de disfunción cetebral fueron diestros en su totalidad (DH en un $75 \%$ y MA en un 25\%).

\section{COMENTARIO}

Las desvirtuadas hipótesis de Samuel Orton acerca de la relación entre dislexia y organización funcional hemisférica cerebral han vuelto a cobrar relevancia con las recientes investigaciones acerca del probable origen disgenésico cerebral de la dislexia, en particular los traba. jos de A. Galaburda sobre organización sitoarquitectónica cortical cerebral en disléxicos, y los planteamientos de N. Geschwind acerca de la zurdera, los trastornos inmunológicos y la distorsión citoarquitectónica cortical cerebral por alteración de la migración neuronal en el período prenatal ${ }^{2,12}$.

A nivel clínico, las hipótesis de estos investigadores se ven apoyadas por los hallazgos de frecuencias eleyadas de zurdera en escolares disléxicos. Desde un punto de vista explicativo, estos niños presentarían alteraciones en la organización cerebral, especificamente de las regiones con participación en las funciones lingü ísticas; tales alteraciones se habrían producido muy tempranamente en el curso del desamollo cerebral (etapa de migración neuronal) y ellas se expresarían, por una parte, como zurdera, y por otra, como una alteración disléxica. Estos zurdos serían "zurdos patológicos", lo que significa que, si no hubiere mediado el factor adverso tempra. no (durante la migración neuronal), tales niffos serían diestros y leerían normalmente. En otras palabras, su condición diestra, determinada genéticamente, habría alterado. Según Annett, los diestros homocigotos estarian en desventaja desde el punto de vista del riesgo de distorsión citoarquitectónica por influencias adversas tempranas, ya que ia acentuada lateralización hemisférica de las funciones lingüísticas reduciría las posibilidades de compensación por parte del hemisferio menor, en caso de compromiso unilateral. En cambio, los djestros heterocigotos, al tener una menor definición cerebral de las funciones lingǘsticas (tendencia a la representación bilateral), compensarían con mayor éxito el déficit.

En términos de expresión genotípica, podemos asumir que el subgrupo DH de nuestra categorización de lateraliđad corresponde al dies- tro homocigoto genético; el subgrupo $\mathrm{MA}$, al de diestro heterocigoto; el subgrupo $\mathrm{ZH}$, al de zurdo homocigoto; el grupo MB, al de diestros homocigotos genéticos con alteraciones citoarquitectónicas hemisféricas izquierdas, y el sub. grupo A, a cualesquiera de las 3 categorías genéticas, con alteraciones citoarquitectónicas cerebrales bihemisféricas o más difusas.

Si se suma el número de $\mathrm{MB}$ de nuestro tra. bajo (zurdos "patológicos") a los zurdos homogéneos $\mathrm{ZH}$, se obtiene $17,8 \%$ de disléxicos zur. dos, que contrasta espectacularmente con $1,8 \%$ de lectores normales zurdos, y coincide con 19\% de disléxicos zurdos citado por Annett ${ }^{13}$.

El origen disfuncional cerebral de la zurdera $y$ la dislexia se vería apoyado por el hallazgo de abundantes signos menores de disfunción neurologica en los escolares disléxicos MB y A de nuestro estudio. Sin embargo, es importante enfatizar que estos signos neurológicos son inespecíficos; esta característica queda en evidencia al encontrar signos de disfunción cerebral en un 14\% de niños lectores normales.

Finalmente cabe destacar que el hallazgo de todo el espectro de lateralidad en nuestra muestra de escolares disléxicos es una evidencia elocuente del carácter heterogéneo de la dislexia (o, más precisamente, las dislexjas), y constituye un incentivo para proseguir la investigación en este campo.

\section{RESUMEN}

Sobre la base de recientes hipótesis acerca del origen disgenésico cortical cerebral de la dislexia y su relación con zurdería, se estudió la distribución de la lateralidad manual y la frecuencia de signos menores de disfunción cerebral, en un grupo de 56 escolares disléxicos de nivel socioeconómico bajo, comparando con un grupo de 56 lectores normales provenientes de las mismas escuelas. Se encontró una elevada frecuencia de zurdos en la población disléxica, la mayoría de ellos con un patrón de zurdería particular, coincidente con el modelo de "zurdo patológico" de la literatura, y con una alta frecuencia de signos menores de disfunción neurológica. Sobre la base de estos resultados, se discuten los postulados de Galaburda y Geschwind acerca de los trastomos de la citoarquitectónica cerebral como sustrato de la zurdería, alteraciones del lenguaje y dislexia. 


\section{REFERENCIAS}

1. Orton S.T.: Reading, writing and speech problems in children. Norton, New York, 1937.

2. Galobunda A.M. and Kemper T.L.: Cytoarquitectonic abnormalities in developmental dyslexia: a case study. Ann Neluol 1979;6: 94-100.

3. Galaburdo A.: La dysiexia et le développement du cerveau. La Recherche. 1985; 16: 762-769.

4. Gaddes W.H.: Cerebral dominance, handedness and laterality. En W.H. Gaddes (Ed.) Learning Disabilities and Brain Function. New York, Springet-Verlag, 1980 .

5. Annett M.: A model of the inheritance of handedness and cerebral dominance. Nature (London). 1964; 204: 59-60.

6. Annett $M$.: The distribution of manual asymmetry, Brit J Psychol. 1972; 63: 343-358.

7. Annetr $M$ : Genetic and non genetic influences on handedness. Behay Genet 1978; 8: 227-249.

8. Annert $M_{.}$: The right-shift theory of handedness and developmental language problems. Bull Orton Soc 1981 ; 31 : $103-121$.

9. Coren S. and Porac C.: Birth factors and lateralty: effects of birth order, parental age and birth stress on four indices of lateral preference. Behav Genet 1980; 10: 123-138.

10. Sat $z$ P.: Pathological left-handedness: an explanatory note. Cortex. 1972; 8: 121-135.

11. Satz $P_{1}$, Orsini Dl., Sasiow W. and Heny R.: The pathological left-handedness syndrome. Brain and Cognition. 1986; 4: 27-46.

12. Geschwind $N$, and Behan P.: Left-handedness: association with immune disease, migraine and developmental learning disorder. Proc Natl Acad Sci (LSA). 1982; 79:5097-5100.

13. Annett $M$. and Kitshow D.: Lateral preference and skill in dyslexics: implication of the right shift theory, J Child Psychol Psychiat 1984; 25: $357-377$.

14. Peters J.E., Romine J.S. and Dykman R.A.: A special neurological examination of children with learning disabilities. Develop Med Child Neurol $1978 ; 17 \div 63-78$. 